\title{
Drastic Cardiac Reverse Remodeling Following Catheter Ablation in Patients with Atrial Fibrillation and Heart Failure
}

\author{
Takahisa Koi, Naoya Kataoka, Teruhiko Imamura *(D) and Koichiro Kinugawa
}

The Second Department of Internal Medicine, University of Toyama, 2630 Sugitani, Toyama 930-0194, Japan; takahisa.koi@i.softbank.jp (T.K.); nkataoka@icloud.com (N.K.); kinugawa-tky@umin.ac.jp (K.K.)

* Correspondence: teimamu@med.u-toyama.ac.jp; Tel.: +81-764-342-281; Fax: +81-764-345-026 check for

updates

Citation: Koi, T.; Kataoka, N.; Imamura, T.; Kinugawa, K. Drastic Cardiac Reverse Remodeling Following Catheter Ablation in Patients with Atrial Fibrillation and Heart Failure. Medicina 2021, 57, 511. https://doi.org/10.3390/ medicina57050511

Academic Editors: Aron-Frederik Popov and Anton Sabashnikov

Received: 12 April 2021

Accepted: 17 May 2021

Published: 20 May 2021

Publisher's Note: MDPI stays neutral with regard to jurisdictional claims in published maps and institutional affiliations.

Copyright: (c) 2021 by the authors. Licensee MDPI, Basel, Switzerland. This article is an open access article distributed under the terms and conditions of the Creative Commons Attribution (CC BY) license (https:// creativecommons.org/licenses/by/ $4.0 /)$.
Abstract: In the management of atrial fibrillation in patients with heart failure, rate control is recommended, whereas the implication of rhythm control remains controversial. We experienced a 65-year-old man who had compensated heart failure due to hypertensive heart disease and atrial fibrillation with well-controlled heart rate $(<100 \mathrm{bpm})$. At three months following the catheter ablation procedure, the left ventricular ejection fraction improved from $40 \%$ up to $65 \%$. The implication of rhythm control using catheter ablation in improving cardiac reverse remodeling should be validated in large-scale clinical studies.

Keywords: hemodynamics; reverse remodeling; arrhythmia

\section{Introduction}

There is a considerable association between atrial fibrillation and heart failure. The presence of one increases the likelihood of another [1]. Atrial fibrillation is an independent predictor of mortality in patients with heart failure [2].

Atrial fibrillation facilitates the progression of heart failure [3]. The incremental heart rate is associated with tachycardia-induced cardiomyopathy. Tachycardia or bradycardia decreases cardiac output and deteriorates hemodynamics. The loss of atrial kick diminishes left ventricular filling. The existence of atrial fibrillation activates the renin-angiotensinaldosterone system.

The first-line therapy for those with heart failure and atrial fibrillation is a rate control using beta-blocker in combination with other medications if necessary [4]. The rhythm control is recommended in selected patients, using antiarrhythmic medications or catheter ablation [5]. However, its impact on cardiac function remains unknown. We experienced a heart failure patient who achieved drastic cardiac reverse remodeling following catheter ablation to treat atrial fibrillation.

\section{Care Report \\ 2.1. Before Admission}

A 65-year-old man with a history of two times of hospitalizations due to worsening heart failure and long-standing untreated hypertension admitted to our institute for further treatment.

At his first decompensated heart failure, bisoprolol and amiodarone were initiated for his systolic heart failure (45\% of left ventricular ejection fraction) and sustained ventricular tachycardia. Following the discharge, persistent atrial fibrillation developed.

At his second decompensated heart failure, the etiology was diagnosed as hypertensive heart disease according to the findings of endomyocardial biopsy with no myocardial fibrosis and heterogeneous myocardium and $25 \%$ of left ventricular ejection fraction as well as no significant coronary artery stenosis demonstrated by coronary angiography. His heart rate was controlled below $100 \mathrm{bpm}$ during the observational period. 


\subsection{On Admission}

His heart failure was relatively compensated with New York Heart Association functional class II using $100 \mathrm{mg}$ of amiodarone, $5 \mathrm{mg}$ of enalapril, $5.0 \mathrm{mg}$ of bisoprolol, $25 \mathrm{mg}$ of spironolactone, $60 \mathrm{mg}$ of azosemide, and $20 \mathrm{mg}$ of furosemide which was added at the second decompensated heart failure re-admission thoroughly. $2.5 \mathrm{mg}$ of warfarin also administrated. Body height was $166 \mathrm{~cm}$ and body weight was $65 \mathrm{~kg}$ (body mass index 23.7). Blood pressure was $100 / 74 \mathrm{mmHg}$ and pulse rate was $86 \mathrm{bpm}$ (irregular). An institutional review board approved the method (R2015154, 11 April 2016) and an informed consent was obtained from the patient.

The estimated glomerular filtration ratio was $57 \mathrm{~mL} / \mathrm{min} / 1.73 \mathrm{~m}^{2}$, plasma B-type natriuretic peptide was $72 \mathrm{pg} / \mathrm{mL}$, and hemoglobin A1c was $6.7 \%$. Chest X-ray showed $46 \%$ of cardiothoracic ratio without obvious pulmonary congestion (Figure 1A). An electrocardiogram showed $91 \mathrm{bpm}$ with atrial fibrillation (Figure 1B). Transthoracic echocardiography showed $50 \mathrm{~mm}$ of left ventricular end-diastolic diameter, $44 \mathrm{~mm}$ of left atrial diameter, and $40 \%$ of left ventricular ejection fraction calculated using a modified Simpson's method (Figure 1C).

A.

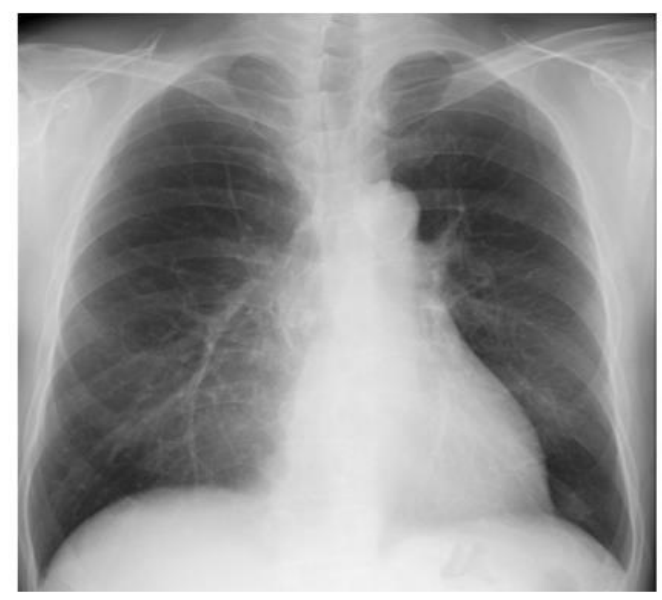

C.

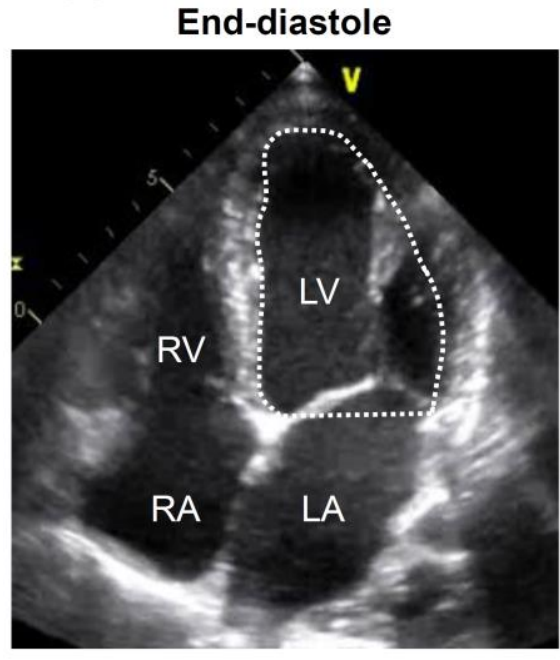

B.

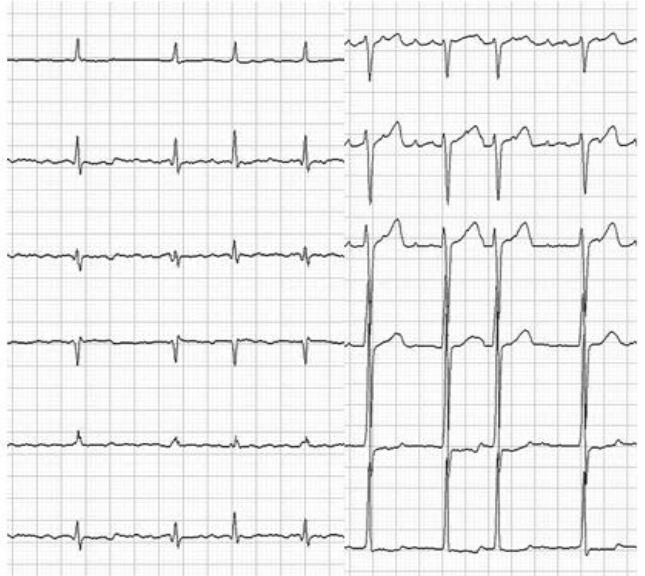

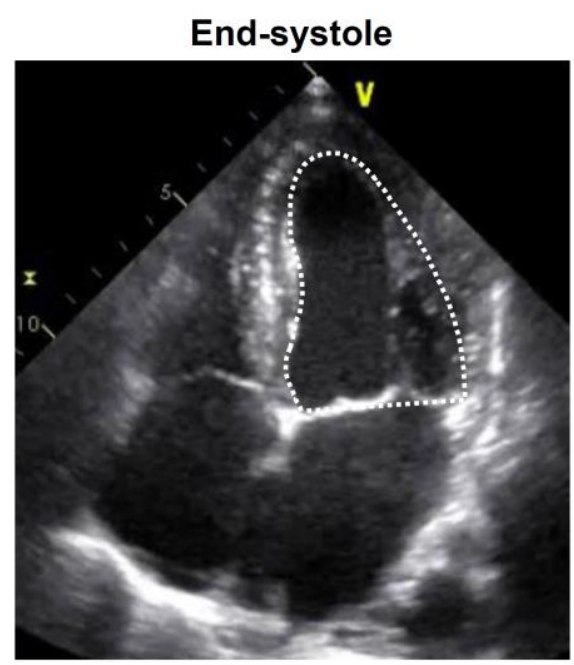

Figure 1. Chest $\mathrm{X}$-ray showing $47 \%$ of cardiothoracic ratio (A), electrocardiogram showing atrial fibrillation (B), and transthoracic echocardiography showing reduced left ventricular ejection fraction on admission (C) on admission. LA, left atrium: LV, left ventricle; Ao, ascending aorta; RA, right atrium; RV, right ventricle. 


\subsection{In-Hospital Course}

Catheter ablation for persistent atrial fibrillation under general anesthesia was performed using the 3-dimensional mapping system (CARTO 3, Biosense Webster, Diamond Bar, CA, USA). Left atrial voltage mapping using a multipolar catheter (PentaRay, Biosense Webster, Irvine, CA, USA) showed healthy electrical voltage except for in the antrum of left inferior pulmonary vein and left atrial septum (Figure 2A). First, the circular electrical isolation of left pulmonary veins was performed. Next, the posterior wall electrical isolation was completed with the ablation for the right pulmonary veins bottom, anterior, and roof lines (Figure 2B). Following the catheter ablation procedure, the patient was discharged on day 5 with a recovery to sinus rhythm (Figure 3A).

A.
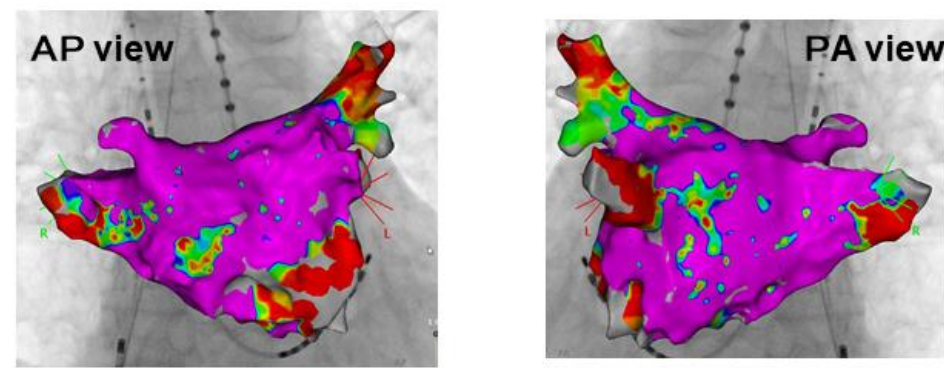

B.
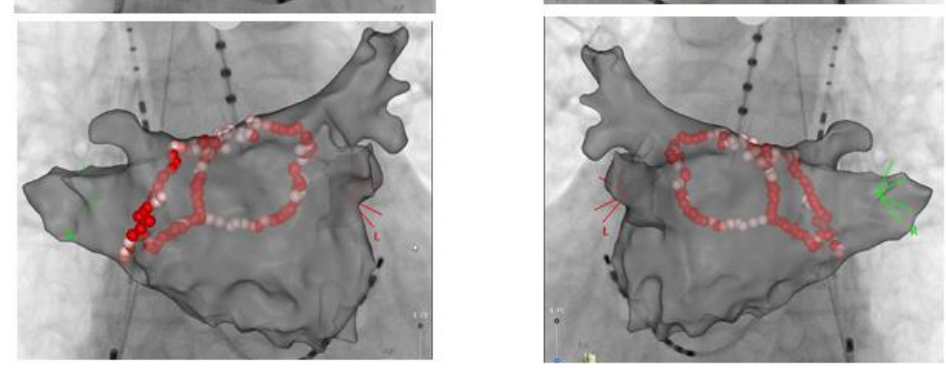

Figure 2. Left atrial voltage mapping (A) and ablation lines (B). AP, Anterior Posterior; PA, Posterior Anterior.

\subsection{Three-Month Follow-Up}

Following the index discharge, anti-heart failure agents remained unchanged. The post-procedural course was uneventful without any adverse events including the recurrence of atrial fibrillation. The patient was evaluated for AF recurrence by a clinical interview, electrocardiogram, and portable electrocardiograph (OMRON Corp., Kyoto, Japan) every month. New York Heart Association functional class was I with $21 \mathrm{pg} / \mathrm{mL}$ of plasma B-type natriuretic peptide at three-month follow-up. Transthoracic echocardiography showed $41 \mathrm{~mm}$ of left atrial diameter and $53 \mathrm{~mm}$ of left ventricular end-diastolic diameter (Figure 3B). Of note, left ventricular ejection fraction improved up to $65 \%$ without any wall motion asynergy. Chest X-ray showed $45 \%$ of cardiothoracic ratio (Figure 3C). 
A.

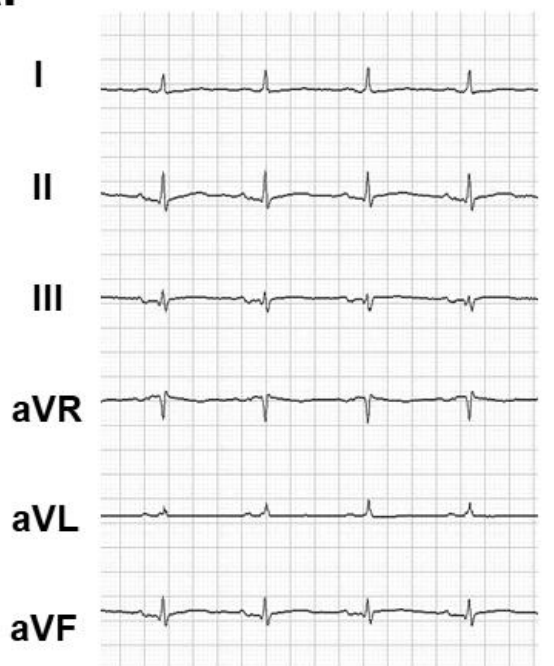

B.
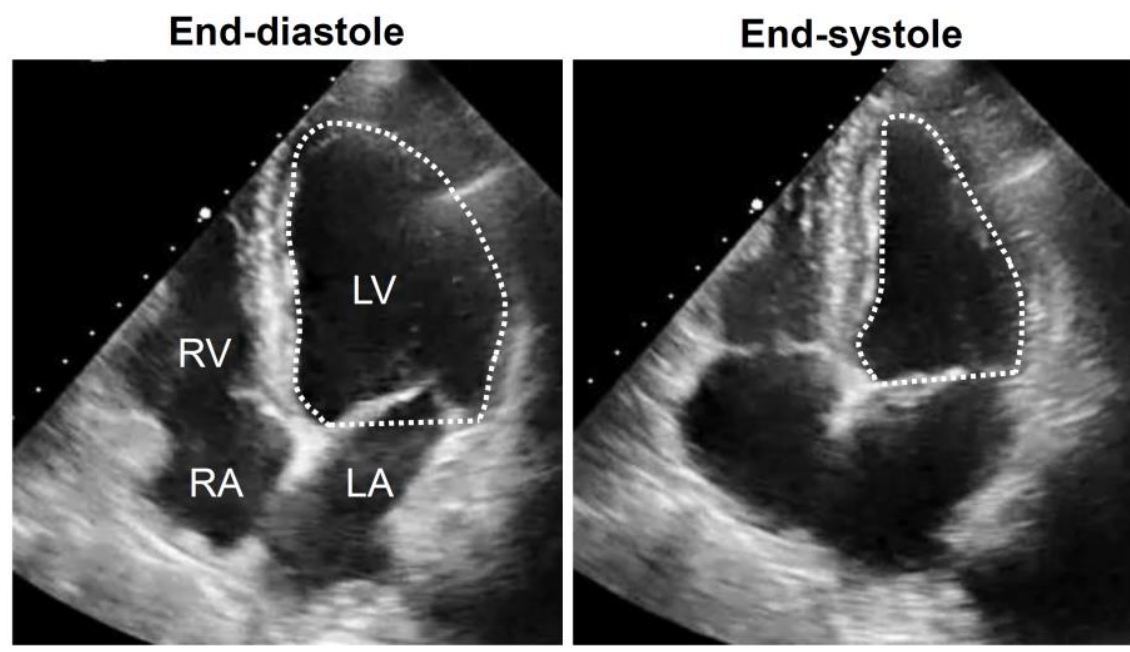

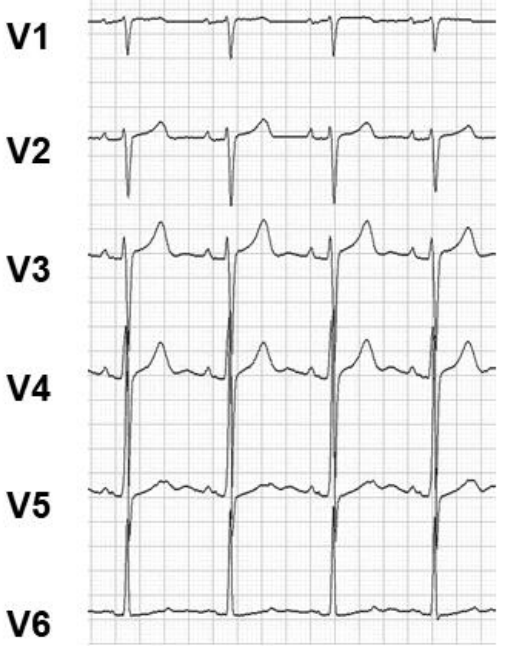

C.

Figure 3. Electrocardiogram at index discharge showing sinus rhythm (A). Transthoracic echocardiography showing $65 \%$ of left ventricular ejection fraction (B) and chest X-ray with $45 \%$ of cardiothoracic ratio (C) obtained at three months following catheter ablation.

\section{Discussion}

\subsection{Atrial Fibrillation and Heart Failure}

Heart failure increases both preload and afterload of the left atrium and progresses its' remodeling, causing the development of atrial fibrillation [1]. The existence of atrial fibrillation reduces ventricular filling due to the lack of atrial kick and stimulates the reninangiotensin-aldosterone system despite heart rate is well controlled [3]. In this patient, as heart failure progresses, atrial fibrillation occurred. The new-onset atrial fibrillation might have triggered the second decompensated heart failure episode.

\subsection{Indication of Rhythm Control}

The first-line to manage atrial fibrillation in patients with heart failure is rate control [4]. If hemodynamics is unstable due to uncontrolled heart rate, electrical cardioversion is considered. Heart rate was well controlled in our patient using a maximum dose of bisoprolol. Given his well-control heart rate, tachycardia-induced cardiomyopathy was excluded. The patient repeated decompensated heart failure against rate control therapy, and we decided to perform rhythm control. 
The Atrial Fibrillation and Congestive Heart Failure (AF-CHF) trial was the first randomized trial that compared the prognostic implication of rhythm control with drug therapy versus rate control (with beta-blockers) in patients with atrial fibrillation and systolic heart failure [6]. The rhythm control strategy did not have a statistically significant advantage over the rate control strategy in improving cardiovascular death. Given the finding, rhythm control using medication is not routinely recommended in patients with atrial fibrillation and heart failure.

Recently, the Cather Ablation for Atrial Fibrillation with Heart Failure (CASTLE$\mathrm{AF}$ ) trial demonstrated the superiority of catheter ablation over the conventional medical therapy for rate or rhythm control in improving all-cause mortality and worsening heart failure in selected patients with atrial fibrillation and systolic heart failure [5]. Given a recent onset of atrial fibrillation and less remodeled left atrium in our patient as well as recent cumulating evidence, we performed catheter ablation.

\subsection{Implication of Rhythm Control on Cardiac Reverse Remodeling}

Our patient experienced a drastic improvement in left ventricular ejection fraction (approximately 25\% increase). Given that guideline-directed medical therapy could not prevent his repeated heart failure recurrences, a dominant cause of his cardiac reverse remodeling would be AF ablation. Such an extreme cardiac reverse remodeling would improve patients' quality of life and exercise capacity as well as preventing recurrent heart failure. His left ventricular end-diastolic diameter slightly enlarged after AF ablation, probably because of the prolonged diastolic duration by decreased heart rate with converted sinus rhythm. Several small studies also found an approximately $10 \%$ increase in left ventricular ejection fraction [7]. The detailed mechanism warrants further studies, but the normalized atrial kick might have reduced the required potential energy. Amelioration of atrial fibrillation might have deactivated the renin-angiotensin-aldosterone system.

Further analyses are required for optimal patient selection [5]. Shorter heart failure duration, non-ischemic etiology with a less myocardial scar, no recurrence of atrial fibrillation, like our patient, might be keys to improvement in cardiac function following catheter ablation. Less left atrial fibrosis, indicated by the existence of healthy electrical voltage in the whole left atrial field in our patient, would also be another key to the incremental left atrial kick that contributes left ventricular reverse remodeling [8]. Cardiac magnetic resonance image analysis, although we did not perform it, would be useful to quantify the left atrial fibrosis and seek optimal patients. Although accurate detection of AF recurrence needs continuous monitoring for heart rhythm, our patient did not receive cardiovascular implantable electronic device implantation, leading to the limitation of this study [9]. Obesity is known as a risk factor of AF recurrence after ablation, but the patient's body mass index was not so high [10]. Glycemic homeostasis and insulin resistance that we did not evaluate may have affected ventricular reverse remodeling [11].

\section{Conclusions}

In conclusion, a rhythm control of atrial fibrillation using catheter ablation would achieve drastic cardiac reverse remodeling in carefully selected patients with systolic heart failure.

Author Contributions: T.K., data collection; N.K., concept; T.I., writing draft; K.K., supervise. All authors have read and agreed to the published version of the manuscript.

Funding: This research received no external funding.

Institutional Review Board Statement: The study was conducted according to the guidelines of the Declaration of Helsinki and approved by the University of Toyama Editorial Board (11 April 2016, R2015154).

Informed Consent Statement: Informed consent was obtained from the patient. 
Data Availability Statement: Data are available from the corresponding author upon reasonable request.

Conflicts of Interest: The authors declare no conflict of interest.

Disclosure: TI receives grant support from JSPS KAKENHI: JP20K17143. NK receives grant support from JSPS KAKENHI: JP19K20724. Other authors have no statements.

\section{References}

1. Cha, Y.M.; Redfield, M.M.; Shen, W.K.; Gersh, B.J. Atrial fibrillation and ventricular dysfunction: A vicious electromechanical cycle. Circulation 2004, 109, 2839-2843. [CrossRef] [PubMed]

2. Carson, P.E.; Johnson, G.R.; Dunkman, W.B.; Fletcher, R.D.; Farrell, L.; Cohn, J.N. The influence of atrial fibrillation on prognosis in mild to moderate heart failure. The V-HeFT Studies. The V-HeFT VA Cooperative Studies Group. Circulation 1993, 87, 102-110.

3. Pozzoli, M.; Cioffi, G.; Traversi, E.; Pinna, G.D.; Cobelli, F.; Tavazzi, L. Predictors of primary atrial fibrillation and concomitant clinical and hemodynamic changes in patients with chronic heart failure: A prospective study in 344 patients with baseline sinus rhythm. J. Am. Coll. Cardiol. 1998, 32, 197-204. [CrossRef]

4. Joglar, J.A.; Acusta, A.P.; Shusterman, N.H.; Ramaswamy, K.; Kowal, R.C.; Barbera, S.J. Effect of carvedilol on survival and hemodynamics in patients with atrial fibrillation and left ventricular dysfunction: Retrospective analysis of the US Carvedilol Heart Failure Trials Program. Am. Heart J. 2001, 142, 498-501. [CrossRef] [PubMed]

5. Marrouche, N.F.; Brachmann, J.; Andresen, D.; Siebels, J.; Boersma, L.; Jordaens, L.; Merkely, B.; Pokushalov, E.; Sanders, P.; Proff, J.; et al. Catheter Ablation for Atrial Fibrillation with Heart Failure. N. Engl. J. Med. 2018, 378, 417-427. [CrossRef] [PubMed]

6. Roy, D.; Talajic, M.; Nattel, S.; Wyse, D.G.; Dorian, P.; Lee, K.L.; Bourassa, M.G.; Arnold, J.M.O.; Buxton, A.E.; Camm, A.J.; et al. Rhythm Control versus Rate Control for Atrial Fibrillation and Heart Failure. N. Engl. J. Med. 2008, 358, 2667-2677. [CrossRef] [PubMed]

7. Anselmino, M.; Matta, M.; D'Ascenzo, F.; Bunch, T.J.; Schilling, R.J.; Hunter, R.J. Catheter ablation of atrial fibrillation in patients with left ventricular systolic dysfunction: A systematic review and meta-analysis. Circ. Arrhythm Electrophysiol. 2014, 7, 1011-1018. [CrossRef] [PubMed]

8. Kirstein, B.; Neudeck, S.; Gaspar, T.; Piorkowski, J.; Wechselberger, S.; Kronborg, M.B.; Zedda, A.; Hankel, A.; El-Armouche, A.; Tomala, J.; et al. Left atrial fibrosis predicts left ventricular ejection fraction response after atrial fibrillation ablation in heart failure patients: The Fibrosis-HF Study. Europace 2020, 22, 1812-1821. [CrossRef] [PubMed]

9. Sardu, C.; Santamaria, M.; Rizzo, M.R.; Barbieri, M.; Di Marino, M.; Paolisso, G.; Santulli, G.; Marfella, R. Telemonitoring in heart failure patients treated by cardiac resynchronisation therapy with defibrillator (CRT-D): The TELECART Study. Int. J. Clin. Pr. 2016, 70, 569-576. [CrossRef] [PubMed]

10. Winkle, R.A.; Mead, R.H.; Engel, G.; Kong, M.H.; Fleming, W.; Salcedo, J. Impact of obesity on atrial fibrillation ablation: Patient characteristics, long-term outcomes, and complications. Heart Rhythm. 2017, 14, 819-827. [CrossRef] [PubMed]

11. Sardu, C.; Santamaria, M.; Funaro, S.; Sacra, C.; Barbieri, M.; Paolisso, P.; Marfella, R.; Paolisso, G.; Rizzo, M.R. Cardiac electrophysiological alterations and clinical response in cardiac resynchronization therapy with a defibrillator treated patients affected by metabolic syndrome. Medicine 2017, 96, e6558. [CrossRef] [PubMed] 